\title{
Heavy Element Nucleosynthesis
}

\author{
Mounib F. El Eid ${ }^{1}$ \\ ${ }^{1}$ American University of Beirut, Department of Physics, Beirut, Lebanon
}

\begin{abstract}
This contribution deals with the important subject of the nucleosynthesis of heavy elements in the Galaxy. After an overview of several observational features, the physical processes responsible mainly for the formation of heavy elements will be described and linked to possible stellar sites and to galactic chemical evolution. In particular, we focus on the neutron-capture processes, namely the s-process (slow neutron capture) and the r-process (rapid neutron capture) and discuss some problems in connection with their sites and their outcome. The aim is to give a brief overview on the exciting subject of the heavy element nucleosynthesis in the Galaxy, emphasizing its importance to trace the galactic chemical evolution and illustrating the challenge of this subject.
\end{abstract}

\section{Introduction}

Elements formation and their evolution is linked to stellar evolution. Fusion reactions can synthesize elements up to Iron, which is the branching point between fusion and fission. Beyond iron, the synthesis of elements is possible only through neutron capture reactions, since neutrons do not experience a coulomb barrier. The main neutron-capture processes are known to be the slow s-process and the rapid r-process. Both of them occur during the late evolutionary phases of stars.

A convenient way of studying the heavy element synthesis is to see their traces in metalpoor halo stars. Their chemical compositions indicates the types of syntheses that happen in the early galaxy. This is a link to "near-field cosmology", or low-red shift universe. The high red-shift universe is not subject directly to observations. But fortunately, we have the primordial nucleosynthesis, the microwave back ground radiation and the Hubble expansion, which help us to draw conclusion about the early universe.

\section{Observations}

There exist now detailed abundance distributions for very metal-poor stars. The presence of heavy elements in these stars indicate the role of a distinct generation of massive stars (second generation?) stars, which were able to synthesize the observed heavy elements. The discovery of radioactive elements (Th, U) in low-metallicity stars helped to date the oldest stars in the Galaxy. In the following, several interesting observations are presented to motivate the theoretical effort explained below. The data in Fig. 1 represent a powerful tool to 

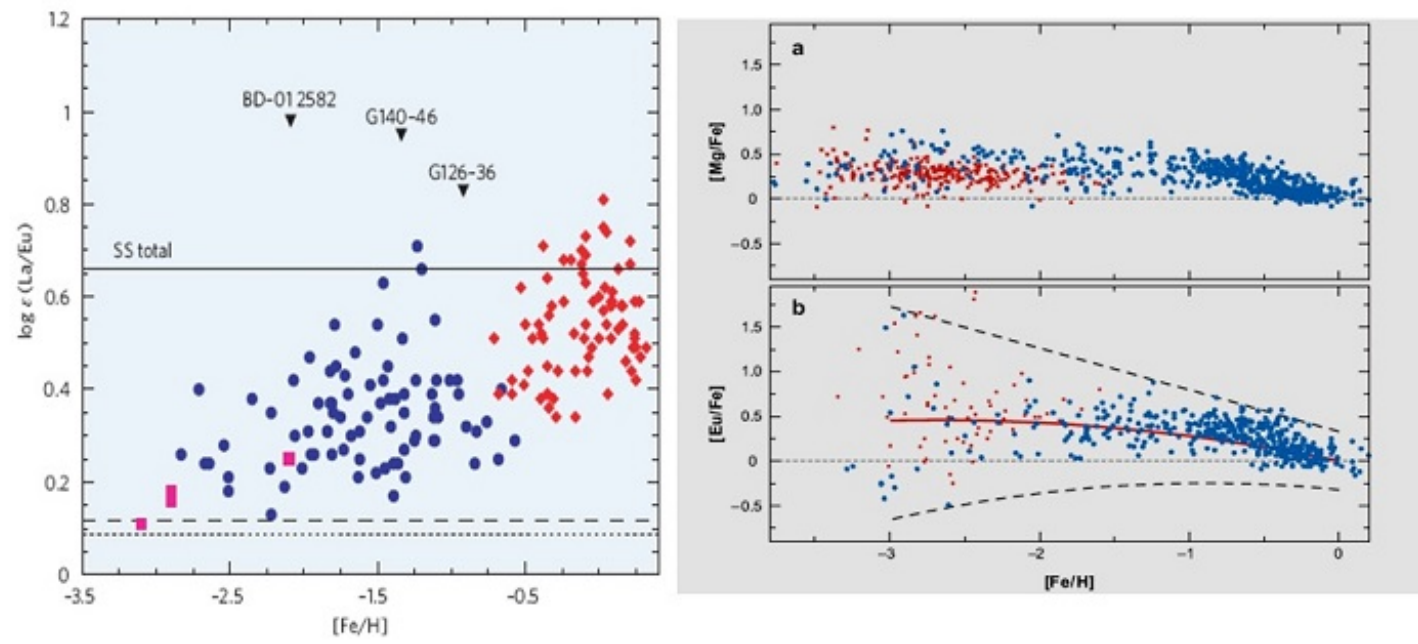

Figure 1. Left panel: Variation of abundances as a function of metallicity. These Figures are taken from [15]. Right panel: Abundances of $\mathrm{Mg}$ and $\mathrm{Eu}$ compared to $\mathrm{Fe}$ as a function of $[\mathrm{Fe} / \mathrm{H}]$. Note that quantities like $[\mathrm{Fe} / \mathrm{H}]$ denote a logarithmic scale, for example $[\mathrm{Fe} / \mathrm{H}]=\log (\mathrm{Fe} / \mathrm{H})_{\text {star }}-\log (\mathrm{Fe} / \mathrm{H})_{\text {sun }}$. See text for details.

study the chemical evolution of the Galaxy. The left panel of this Figure shows interesting features. The abundance ratio of the Lanthanum (La, being mainly produced by the s-process nucleosynthesis) to Europium (Eu, being mainly produced by the r-process) is shown as a function of metallicity $[\mathrm{Fe} / \mathrm{H}]$ for a variety of halo and disk stars. Note that line drawn near to $\log \epsilon(L a / E u)=0.1$ consistent with a pure r-process pollution, and matches very few metal-poor stars. It is also seen that the $\log \epsilon(L a / E u)$ increases with increasing metallicity, where a significant contribution by the s-process starts already at $[\mathrm{Fe} / \mathrm{H}] \simeq-2.0$. It is still not entirely clear how the s-process became effective at such low metallicity, and which is the role of metal-poor massive stars?. A firm point is a clear presence of neutron-capture (briefly n-capture) elements in the atmospheres of metal-poor star.

The comparison between r-process rich $([\mathrm{Eu} / \mathrm{Fe}] \geq 1.0)$ and r-process poor $([\mathrm{Eu} / \mathrm{Fe}]<1.0)$ stars indicates that the abundances of the n-capture elements with $\mathrm{Z} \geq 56$ (barium and above) are consistent with scaled solar r-process distribution. This is now termed "main r-process". In contrast, the abundances of the light n-capture species (first r-process peak around the element Sr) do not match the solar system pattern, and this is termed "weak r-process", because relatively low neutron density of about $10^{20}$ neutron $/ \mathrm{cm}^{3}$ is needed to produce theses species (see [? ]). The right panel of Fig. 1 shows the abundances of the alpha-element Mg and those of the r-process element $(\mathrm{Eu})$. The abundances of $\mathrm{Mg}$ have little scatter and no significant drift at low metallicity. This may imply a significant production of $\mathrm{Mg}$ during the early phase of the Galaxy contributed by supernova explosions of massive stars, [17]. The downward decrease of $[\mathrm{Mg} / \mathrm{Fe}]$ to the solar value indicates the increase in the iron production by type Ia supernovae (exploding white dwarfs). In contrast, the data for $[\mathrm{Eu} / \mathrm{Fe}]$ exhibit deviations from $[\mathrm{Fe} / \mathrm{H}]=0$ at low metallicity. A lager scatter is found as well. The scatter is attributed (see [15] and references therein) to an early unmixed galaxy, where the supernovae were widely scattered. Moreover, the data suggest that the r-process production was rare in 
Table 1. Summary of some characteristics of the s-process and r-process

\begin{tabular}{|c|c|c|}
\hline & s-process & r-process \\
\hline Neutron density $\left[\mathrm{cm}^{-3}\right]$ & $10^{7}-10^{11}$ & $10^{20}-10^{30}$ \\
\hline Astrophysical Site & AGB Stars, Massive Stars & $\begin{array}{l}\text { Supernova type II } \\
\text { Neutron star mergers }\end{array}$ \\
\hline Abundances correlated with & neutron capture cross section & beta-decay lifetimes, n-separatio \\
\hline Neutron source & ${ }^{13} \mathrm{C}(\alpha, n){ }^{16} \mathrm{O},{ }^{22} \mathrm{Ne}(\alpha, n){ }^{25} \mathrm{Mg}$ & begin with neutrons \& protons \\
\hline Temperature range & $\mathrm{T}=(1.0-3.5) \times 10^{8} \mathrm{~K}, \mathrm{~T} \geq 10^{9} \mathrm{~K}$ & $\mathrm{~T}=(1-10) \times 10^{9} \mathrm{~K}$ \\
\hline Basic Characteristic & Secondary process & Primary process \\
\hline
\end{tabular}

the early Galaxy, and only few stars produced elements such as Eu. It seems that in thye earely galaxy, there was no strong coupling between the production of $\mathrm{Mg}$ and $\mathrm{Fe}$ and $\mathrm{Eu}$. Note that the downward decrease of $[\mathrm{Eu} / \mathrm{Fe}]$ to the solar value is again due to the increasing iron production by type Ia supernovae.

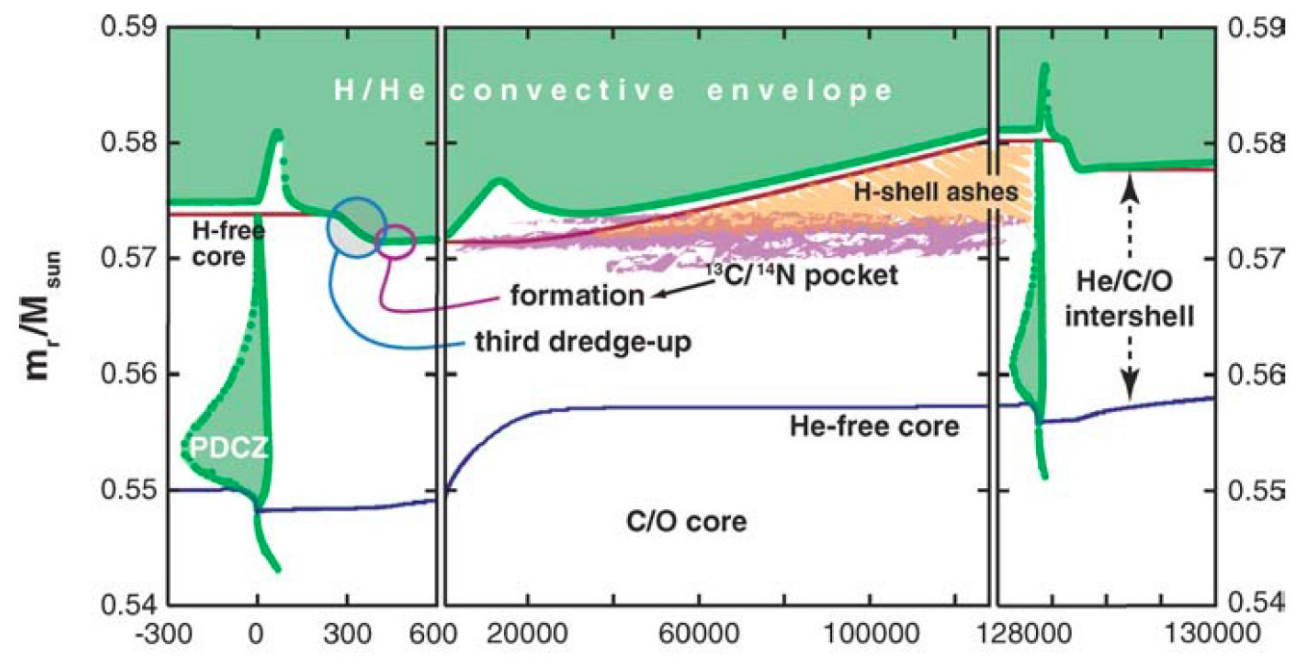

Figure 2. Structure of an AGB star during thermally pulsating phase according to [8] in case of a $2 \mathrm{M}_{\odot}$ star of initial solar-like composition. The green (or darker) areas are convective zones. See text for details

\section{Basic comments on the s-process and r-process}

In Table 1, a comparison between the s-process and the r-process is given. in case of the s-process, a temperature range of $(1.0-3.5) \times 10^{8} \mathrm{~K}$ is needed in core He-burning of massive 
stars $\left(\mathrm{M} \geq 15 \mathrm{M}_{\odot}\right)$, or during the thermal pulses of AGB stars, while $\mathrm{T} \geq 10^{9} \mathrm{~K}$ is needed during shell carbon-burning in massive stars $([6])$. The temperature range for the r-process is higher whether in neutron star mergers, or in an exploding supernova, where it starts near the edge of the newly formed neutron star. In this scenario, the iron-group elements are synthesized in situ as explained below. Regarding the neutron sources, those for the s-process are: ${ }^{13} \mathrm{C}(\alpha, n){ }^{16} \mathrm{O}$ in AGB stars, while ${ }^{22} \mathrm{Ne}(\alpha, n)^{25} \mathrm{Mg}$ becomes effective in massive stars, and could be activated in massive AGB stars (see [8] concerning this point)

\subsection{Comments on the s-process}

Basically, the s-process is a flow concept process controlled by slow neutron-capture reactions with neutron density in the range $10^{7}-10^{11}$ neutrons $/ \mathrm{cm}^{3}$. The produced neutrons thermalize very quickly by s-wave scattering and can be described by Maxwell-Boltzmann distribution. Due to the relatively low neutron density, any unstable nucleus created by n-capture will

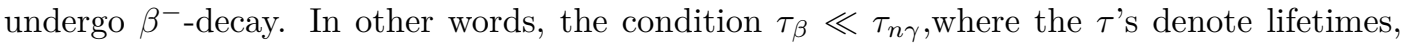
is fulfilled meaning that beta-decay dominates the flow, so the s-process path remain close to the valley of beta-stability (for example see Fig. 9.13 in the text by [14]). However, if the neutron density is high enough like, $10^{11}$ neutrons $/ \mathrm{cm}^{3}$, then branching at an unstable nucleus is possible. An example dealing with Zr-isotopes is given in [8].

\subsection{Comments on the r-process}

The r-process is also based on a flow concept (a review is given by [5]). But in contrast with the s-process, it is a primary process in the Galaxy, since it creates its own seed nuclei. A basic feature of this process is the very high neutron density as seen in table 1. Consequently, the condition $\tau_{n \gamma} \ll \tau_{\beta}$ is fulfilled in this case, and the neutron capture reactions dominate the flow but not at all the time. Due to the fast addition of neutrons, neutron-rich nuclei are formed far from the valley of beta-stability, so that the neutron binding energy decreases and tends to approach the neutron drip line. For a given temperature $\mathrm{T}$ and neutron density an equilibrium between the $(\mathrm{n}, \gamma)$ and $(\gamma, \mathrm{n})$ reactions becomes possible, which is called waiting points for each isotopic chain with proton number Z. This behavior is seen in the Fig. 9.13 in the text by [14]. In particular, the neutron magic nucleus ${ }^{130} \mathrm{Cd}$ with $\mathrm{N}=82$ is a special waiting point for the r-process with a $\beta^{-}$-lifetime of $(127 \pm 2) \mathrm{ms}$ according to the RIKENresults [12]. This means the abundances resulting from the r-process are correlated with the beta decay life times and neutron separation energy in contrast with those from s-process which are correlated with the neutron capture cross section.

\section{Stellar Sites}

The stellar sites of the heavy element synthesis are not easy to describe. In the following we describe briefly possible sites of the s-process and the r-process. No attempt is made to describe the secondary p-process producing proton-rich nuclei bypassed by the s-process (see review by [13].

\section{1 s-process stellar site}

As mentioned in Sect. 2, the s-process started later in the Galaxy, because it is a secondary process which needs iron seed nuclei. The main component of this process comprising nuclei 
with mass numbers $\mathrm{A} \geq 90$ is produced during the thermally pulsing AGB (TP-AGB) stars in the mass range (1-8) $\mathrm{M}_{\odot}$. The dominant contribution matching the solar pattern distribution comes from mass range roughly $(1-3) \mathrm{M}_{\odot}([3])$.

Careful modeling of TP-AGBs is required, in particular to understand the formation of the carbon-rich stars and the production of the main neutron source for the s-process ${ }^{13} C(\alpha, n){ }^{16} O$. This is highly dependent on the mixing of protons from the convective envelope into the helium and carbon rich layers during a thermal pulse. A useful review on general properties of AGB stars is given by ([8], [16], [9]). Fig. 2 shows two successive thermal pulse during the AGB phase of a $2 \mathrm{M}_{\odot}$ star. The crucial issue in such calculations is the treatment of the so called third dredge up (TDU). On ground of the usual treatment of convection instability according to the well-known Schwarzschild criterion, the extension of the H-rich convective envelope after the He-shell flash (or thermal pulse) is not deep enough in order to inject protons into the carbon enriched layers. This is requited to activate the neutron source for the s-process nucleosynthesis, which is created by the reaction ${ }^{12} C(p, \gamma){ }^{13} N\left(\beta^{+}\right){ }^{13} C(\alpha, n){ }^{16} O$. A key problem is to get the sufficient amount of ${ }^{13} \mathrm{C}$ in order to explain the s-process abundances in the Galaxy ([19]). In [19], an important point is emphasized that the understanding of the s-process should not be restricted to matching the solar s-process abundances, but should concern with aspects of the chemical evolution in the Galaxy as well.

The production of ${ }^{13} \mathrm{C}$ is linked tightly to the to the TDU. Several effects play a role in fixing the extension of the TDUP, like extra diffusion of protons termed "overshooting" (see discussion in ([8]), [2], [4]), rotationally-induced mixing ([11]), or a recent attempt considering the effect of mixing by magnetic buoyancy in the framework of magnetohydrodynamics ([20]). More work is needed to better understand mixing processes during the AGB phases of stars.

\section{2 r-process stellar site}

The favored site of the r-process nucleosynthesis was the high entropy wind (HEW) in core collapse supernovae (CCSN). A basic scenario is ([10], [21]) that the streaming out neutrinos from the forming neutron star represent a neutrino-driven wind starting near the surface of the newly forming neutron star with a flow of neutrons and protons. In a temperature range $\mathrm{T}=(6-10) \times 10^{9} \mathrm{~K}$, the neutrons and protons combine to form alpha-particles, but with excess of neutrons. When the temperature drops to the range $\mathrm{T}=(3-6) \times 10^{9} \mathrm{~K}$, nuclei in the iron group are able to form, which serve as seed nuclei for the r-process. This is called alpha freeze out. Upon further cooling to the range $\mathrm{T}=(1-3) \times 10^{9} \mathrm{~K}$, the r-process becomes effective.

However the role of neutron star mergers has changed the picture significantly (see reviews by [18], [1]). It seems now that neutron star mergers constitute the major cite of the heavy element production by the r-process, and the only source of elements beyond lead and bismuth [18]. The remarkable insight was that the neutron star mergers were identified with short duration of Gamma-ray Bursts via their IR afterglow, to be explained by the opacities of heavy element beyond the iron group.

Does this discussion mean that the core collapse supernovae (CCSN) are removed from being the site of any r-process?. An answer to this question is not straightforward. For example, the behavior of $[\mathrm{Eu} / \mathrm{Fe}]$ shown in Fig. 1 with its large scatter at low metallicities may reveal the role of early CCSN of massive stars. It seems that the large scatter of $[\mathrm{Eu} / \mathrm{Fe}]$ indicates production with low event rate with high amount of r-process to explain the solar abundance, and a lack of mixing into the interstellar medium of these products steaming from CCSN. In short, the role of CCSN contributing to the r-process cannot be excluded especially at low metallicities. 
The signature of the r-process is important in halo stars, since they are the oldest objects in the Galaxy, providing important insight about its early evolution. A well-studied halo star is CS22892-052 by [15] having $[\mathrm{Fe} / \mathrm{H}]=-3.0$. A striking finding is the agreement between the abundances of this star and the solar r-process abundances for $\mathrm{Z} \geq 56$ (that is starting from $\mathrm{Ba}$ ). But no agreement for range $\mathrm{Z}=40-50$ (first $r$-process peak) was found. It is beyond the scope of this contribution to discuss this issue. It seems that the r-process was not happening the same way all the time as it has been thought. There is an incomplete (or weak) component similar to the s-process. More work is needed to understand the nature site of this component.

\section{Brief Summary}

The heavy elements and their isotopes are mainly produced by the s-process and the r-process and to less extent by the p-process. They trace back the chemical evolution of the galaxy, thus they represent a link to the low red-shift universe. Many details of these processes are still not understood, especially the physical conditions under which they operate. In case of the s-process the treatment of mixing on the AGB phase is rather challenging and needs further elaboration. Eventually three dimensional simulations are desired to at least constraint the one-dimensional description. In case of the r-process occurring in core collapse supernovae, the modeling of the explosion is still challenging, especially in light of the newest observations of the radioactive element ${ }^{44} \mathrm{Ti}$ in the supernova remnant Cassiopeia $A$ as observed by the NuStar team ([7]. The site of the r-process has received a new insight after the discovery of neutron star merger GW180817 by the LIGO and VIRGO collaboration.

Acknowledgment: The author thanks the referee for careful reading and useful comments.

\section{References}

[1] Baiotti, L., Rezzola, LK., Rep. Prog. Phys. 80, 096901 (2017)

[2] Bisterzo, S., J. Phys. Conf. Ser., 665, 012023 (2016)

[3] Busso, M. et al., Ann. rev. A\&A 37, 239 (1999)

[4] Cristallo, R. et al., APJ, 833, 181 (2016)

[5] Cowan, J.J., Thielemann, F-K., Phys. Today 57, 47 (2004)

[6] El Eid, M.F., et al. Space Sc. Rev. 147, 1 (2009)

[7] Grefenstette, R.W. et al., Nature, 506, 339 (2014)

[8] Herwig, F., Ann. rev. A\&A 43, 435 (2005)

[9] Karalkas, A., Lattanzio, J.c., PASA 31, e030 (2014)

[10] Kratz, K.L., AIP Cof. Proc., 1065, 109 (2015)

[11] Langer, N. et al. A\&A 346, 37L (1999)

[12] Lorusso, G. et al., Phys. Rev. Lett., 114, 192501 (2015)

[13] Meyer, B.S., Ann. rev. A\&A 32, 152 (1994)

[14] Rolfs, C., Rodney, W., Cauldrons in the Cosmos,(Chicago University press, Chicago, 1988) p. 472

[15] Sneden, C. , et al., Ann. rev. A\&A 46, 241 (2008)

[16] Straniero, O. et al., Nucl. Phys. A, 777, 311 (2006)

[17] Thielemann, F.-K et al. , Mem. Soc. Aston. Ital. 71, 481 (2000)

[18] Thielemann, F.K. et al., Ann. Rev. Nucl. Part. Sci., 67, 253 (2017)

[19] Trippella, O., et al., ApJ 787, 41 (2014) 
[20] Trippella, O., et al., ApJ 818, 125 (2016)

[21] Woosley, S. Janka, T., Nature phys, 1, 147 (2005) 